\title{
Investigation of the frequency and clinical manifestations of Wegener vasculitis among other vasculitis patients in rheumatology ward of Vali-asr hospital during the past 10 years
}

\author{
Rabeah Rajabzadeh ${ }^{1}$, Seyed Reza Najafizadeh ${ }^{\text {, }}$, Shafieh Movassaghi ${ }^{1}$, Sara Jalali-Jivan ${ }^{1}$, Abdolrahman Rostamian ${ }^{1,2 *}$ \\ ${ }^{1}$ Department of Rheumatology, Imam Khomeini Hospital, School of Medicine, Tehran University of Medical Sciences, \\ Tehran, Iran. ${ }^{2}$ Rheumatology Research Center, Imam Khomeini Hospital, Tehran University of Medical Sciences, \\ Tehran, Iran.
}

\begin{abstract}
Vasculitis is a rare disease and its prevalence varies according to age, race, time, and geographical location. The need for this study is felt due to the fact that no study has been conducted in Iran to investigate the frequency, clinical, and laboratory symptoms of vasculitis in the considerable population of vasculitis patients. The aim of this study was to evaluate the frequency and clinical signs of granulomatosis with polyangiitis (GPA) patients who were admitted to the rheumatology ward in Valiasr Hospital, Tehran, Iran, over the last 10 years.

In this study, all patients were evaluated with a possible diagnosis of vasculitis having been admitted to the rheumatology ward of Valiasr Hospital over the past ten years. These GPA patients were evaluated for clinical, laboratory, radiological, and pathological findings, as well as for outcome, mortality, and major complications.

Over the last 10 years, 223 vasculitis patients have been admitted to Valiasr Hospital and of these, $87 \%$ were primary vasculitis and $13 \%$ were secondary vasculitis. The highest frequncy was related to GPA $(21.52 \%)$ followed by Behcet's disease. GPA patients were women and men at $52.08 \%$ and $47.91 \%$, respectively. Upper and lower airway involvement and total ear, nose, and throat (ENT) involvement were the most common clinical symptoms in GPA patients.

According to the findings of this study, GPA vasculitis was the most common type of vasculitis and ENT involvements were the most common clinical symptoms in this patient. However, more studies are required to confirm these data in Iran.
\end{abstract}

Keywords: Wegener, Frequency, Vasculitis, Clinical Symptoms

\section{Introduction}

Vasculitis means inflammation and swelling of the blood vessels, which can cause inflammation of the walls of the small vessels resulting in reduced blood flow and blockage of the vessels and lack of blood supply to various tissues and organs of the body. Annually, 10 to 15 out of every one million people get the disease, most of whom are over 50 years of age [1]. Children and young people are also at risk. The disease can be diagnosed by angiography or biopsy (taking a small piece of tissue from the affected area of the body). The symptoms are swelling of blood vessels in the affected part of the body and even rupture and internal bleeding in the same part [2]. There are two types of vasculitis; namely, primary and secondary, which have no underlying cause for the emergence of primary vasculitis, and the cause of secondary vasculitis is rheumatic diseases such as systemic lupus erythematosus
(SLE), and rheumatoid arthritis (RA) which are more prevalent [3].

Granulomatosis with polyangiitis (GPA), based on the American College of Rheumatology (ACR) 1990 definition, is a systemic vascular necrosis of small blood vessels with two of the four findings in urinary sediment, abnormal chest radiological findings, oral ulcers, and secretions discharge $[4,5]$. Clinical symptoms are caused by involvement of the upper airway, lungs, eyes, gastrointestinal tract, heart, kidneys, skin, and spleen. It occurs most often in the 4th and 5th decades of life and is associated with $80 \%$ mortality if left untreated. Inflammation, obstruction, and vascular ischemia have been implicated in the pathophysiology of the disease, but the initiating and accelerating factors remain unclear [4].

Personal non-commercial use only. Rheumatology Research Journal. Copyright $($ C 2020. All rights reserved

*Corresponding Author: Dr Abdolrahman Rostamian, Department of Rheumatology, School of Medicine, Tehran University of Medical Sciences, Tehran, Iran. Email: arostamian@tums.ac.ir,

Received: 09 October 2019; Accepted: 20 October 2019 
With regard to the different epidemiology of rheumatic diseases in different countries, recognizing the diversity and differences in the initial symptoms of the disease is of particular importance and early diagnosis plays a significant role in the prognosis of the disease. The variety of clinical symptoms of GPA causes the patient to refer to different medical specialists. Most often, the onset of clinical symptoms of the disease is related to the head and neck $(75 \%)$ that puts this disease in the differential diagnosis of other diseases in this area, which may initially lead to symptomatic treatment and eventually delay the primary treatment [6].

According to previous studies, vasculitis has important effects on the health of a person, which makes it necessary to study the epidemiology of this disease in different populations. Considering that no study has been conducted in Iran to comprehensively study the epidemiology of this disease, this present research was performed with the aim of determining the frequency of GPA vasculitis. The aim of this study was also to evaluate the frequency and clinical signs and symptoms of granulomatosis with polyangiitis patients, among vasculitis patients, admitted over the past 10 years to the rheumatology ward of Valiasr Hospital.

\section{Materials and Methods}

The study population included patients who had been admitted to the rheumatology ward of Valiasr Hospital over the past 10 years with a diagnosis of vasculitis. In this study, patients who had been admitted to the hospital with a possible diagnosis of vasculitis were examined. Diagnosis was initially performed according to the American College of Rheumatology (ACR) in 1990 by the Chapel Hill Consensus Conference (CHCC) criteria [7]. According to the ACR criteria, if the patient meets four criteria: Nasal discharge or oral ulcers, abnormal lung Xray (presence of nodules, cavities, constant infiltration), abnormal urine test (presence of red blood cells or more than 5 red blood cells in each microscopic field under high magnification), the presence of granulomatous inflammation in biopsy, or has at least two criteria with a sensitivity of $88.2 \%$ and specificity of $92 \%$ is classified as granulomatous vasculitis with GPA.

The data were analyzed using a checklist designed by the researchers. This checklist included the following: Sex, age, clinical signs and symptoms (based on history and clinical examination) including upper airway symptoms that are the most common early signs of the disease with epistaxis, nasopharyngeal mucopurulent discharge, painful inflammation of the nose and paranasal sinuses, ear congestion and conductive hearing loss, subglottic stenosis (in effect of active inflammation or scarring), oral ulcers, gingivitis, eardrum chondrites, deformity of the nasal septum that can lead to saddle deformity of the nose, sinuses and orbit. The protocol of the study was approved by the Ethical Committee of Tehran University of Medical Sciences (Code: IR.TUMS.IKHC.REC.1399.040).

\section{Statistical Analysis}

Data analysis was performed using SPSS software (version 25) at descriptive levels to describe the demographic and clinical status of the patients. Quantitative variables were shown as mean \pm standard deviation and categorical variables were shown as percentage. $P$ value $<0.05$ was considered as statistically significant.

\section{Results}

In the present study, first, all vasculitis patients admitted to Valiasr Hospital over the last 10 years were selected. The types of vasculitis in these patients are shown in Table 1. A total of 223 vasculitis patients were referred over the last 10 years, 48 of whom were GPA patients. The mean age and disease duration were $36 \pm 14.45$ years and $26.3 \pm 15.4$ months, respectively. In 48 GPA patients, 25 (52.08\%) were female and $23(47.91 \%)$ were male. The mean age of female and male was $35.52 \pm 15.39$ and $36.53 \pm 13.63$ years, respectively.

Table 1. Frequency of vasculitis type in vasculitis patients referred to Vali-Asr Hospital

\begin{tabular}{|c|c|}
\hline Type of vasculitis & N (\%) \\
\hline GP & $48(21.52)$ \\
\hline $\mathrm{BD}$ & $34(15.24)$ \\
\hline Cutaneous Leukocytoclastic & $21(9.41)$ \\
\hline EGPA & $19(8.52)$ \\
\hline BD-Vasculitis & $15(6.72)$ \\
\hline Takayaso & $14(6.27)$ \\
\hline PAN & $12(5.38)$ \\
\hline RA-vasculitis & $12(5.38)$ \\
\hline GCA & $8(3.58)$ \\
\hline Small vessel vasculitis & $8(3.58)$ \\
\hline Medium vessel vasculitis & $6(2.6)$ \\
\hline Undifferentiated vasculitis & $5(2.24)$ \\
\hline SLE-vasculitis & $3(1.34)$ \\
\hline HSP & $2(0.89)$ \\
\hline Cutaneous PAN & $2(0.89)$ \\
\hline Small-medium vessel vasculitis & $2(0.89)$ \\
\hline MPA & $2(0.89)$ \\
\hline Sjogren's vasculitis & $2(0.89)$ \\
\hline Retin vasculitis & $1(0.44)$ \\
\hline Brainstem vasculitis & $1(0.44)$ \\
\hline Good Pasture & $1(0.44)$ \\
\hline Large vessel vasculitis & $1(0.44)$ \\
\hline
\end{tabular}




\begin{tabular}{cc}
\hline Type of vasculitis & N (\%) \\
\hline RA-sjogren's vasculitis & $1(0.44)$ \\
Scleroderma vasculitis & $1(0.44)$ \\
Urticaria & $1(0.44)$ \\
Dermatomyositis-vasculitis & $1(0.44)$
\end{tabular}

$\mathrm{GP}=$ Granulomatosis with polyangitis $; \mathrm{BD}=$ Behcet's disease; EGPA $=$ Eosinophilic Granulomatosis with Polyangiitis, PAN =
Polyarteritis nodosa; $\mathrm{RA}=$ Rheumatoid arthritis; $\mathrm{GCA}=$ Giant cell arteritis; SLE $=$ Systemic lupus erythematosus; HSP $=$ HenochSchönlein purpura; MPA = Microscopic polyangiitis

Out of 223 vasculitis patients, 194 (86.99\%) were primary vasculitis and other cases were secondary vasculitis (Table 2). Most cases of secondary vasculitis were related to RA secondary vasculitis (Table 2).

Table 2. Distribution of primary and secondary vasculitis in vasculitis patients referred to Valiasr Hospital

\begin{tabular}{cc}
\hline Type of vasculitis & N (\%) \\
\hline Primary vasculitis & $194(86.99)$ \\
Secondary vasculitis & RA \\
Drug & $12(5.38)$ \\
SLE & $9(4.03)$ \\
Sjogren's & $3(1.34)$ \\
RA-sjogren's & $2(0.89)$ \\
Scleroderma & $1(0.44)$ \\
Dermatomyositis & $1(0.44)$ \\
\hline
\end{tabular}

RA = Rheumatoid arthritis; SLE $=$ Systemic lupus erythematosus

Table 3 shows the frequency distribution of clinical symptoms in GPA patients. Upper and lower airway involvement and total ear, nose and throat (ENT) involvement were the most common clinical symptoms in GPA patients. In contrast, cardiac and neurologic manifestations, fever, and oral ulcers were less common. The radiological findings of the GPA patients are shown in Table 4. Increased thickness of paranasal sinuses, cavitation, and pulmonary nodules were seen in 31.25 ,
27.08 and $25 \%$ of GPA patients, respectively (Table 4). Sixty-eight point seventy-five and $12.5 \%$ of patients were anti-neutrophil cytoplasmic autoantibody (C-ANCA) positive and anti-PR3 positive, respectively, and $8.33 \%$ of patients had a coexistence of C-ANCA and anti-PR3 (Table 5). In total, 35 cases from 48 GPA patients were positive for C-ANCA or anti-PR3.

Table 3. Frequency distribution of clinical symptoms in Wegener vasculitis patients

\begin{tabular}{cc}
\hline Parameter & N (\%) \\
\hline Lower airway involvement & $26(54.16)$ \\
Upper airway involvement & $24(50)$ \\
Cutaneous involvement & $14(2.16)$ \\
Auditory involvement & $12(25)$ \\
Ocular involvement & $11(22.91)$ \\
Articular involvement & $11(22.91)$ \\
Weakness & $10(20.3)$ \\
Fever & $9(18.75)$ \\
Neuropathy & $8(16.66)$ \\
Cardiac involvement & $7(14.58)$ \\
Oral ulcer & $5(10.41)$ \\
\hline
\end{tabular}


Table 4. Radiological findings in Wegener vasculitis patients

\begin{tabular}{cc}
\hline Parameters & $\mathrm{N}(\%)$ \\
\hline Increasing the thickness of the para-nasal sinuses & $15(31.25)$ \\
Pulmonary Cavitation & $13(27.08)$ \\
Pulmonary nodule & $12(25)$ \\
Alveolar hemorrhage & $9(18.75)$ \\
Consolidation & $4(8.33)$ \\
Pleural effusion & $3(6.25)$ \\
Fibrosis & $3(6.25)$ \\
Bronchiectasis & $1(2.08)$
\end{tabular}

Table 5. Frequency distribution of laboratory findings in Wegener vasculitis patients

\begin{tabular}{cc}
\hline Parameter & N $(\%)$ \\
\hline C-ANCA $(+)$ & $33(68.75)$ \\
Anti-PR3 $(+)$ & $6(12.5)$ \\
Coexistence of C-ANCA and Anti-PR3 & $4(8.33)$ \\
\hline
\end{tabular}

C-ANCA = Anti-neutrophil cytoplasmic antibodies; Anti-PR3 = Anti- proteinase 3.

\section{Discussion}

The aim of this current study was to determine the frequency and clinical signs and symptoms of GPA vasculitis patients among other vasculitis patients in the rheumatology ward of Valiasr Hospital over the past 10 years. Of the 223 vasculitis patients studied, GPA had the highest frequency, followed by Behcet's disease and leukocytoclastic vasculitis. It was also observed that $87 \%$ of the cases were primary vasculitis and $13 \%$ were secondary vasculitis. In this regard, in a study by Alvise Berti et al. [8] 58 cases of ANCA (antineutrophil cytoplasmic antibody)-associated vasculitis (AAV) were studied and it was observed that the highest prevalence is first microscopic polyangiitis (MPA), then GPA and Church Strauss. Aksel Thuv Nilsen et al. studied the prevalence of ANCA-related vasculitis in 140 vasculitis patients. The results of their study showed that the highest prevalence was related to GPA, followed by MPA and eosinophilic granulomatosis with polyangiitis (EGPA) [6].

The results of the present study showed that the mean age of GPA patients in men and women was $35.52 \pm 15.39$ and $36.53 \pm 13.63$ years, respectively. Contrary to previous sources and studies, the frequency of women with GPA was higher than in men. Consistent with this finding, in a study by Alvise Berti et al. [8], 58 cases of AAV vasculitis were studied and it was shown that the frequency of women with GPA was higher than in men.

In the present study, upper and lower airway involvement and total ENT involvement were the most common clinical symptoms in GPA patients. In contrast, cardiac and neurologic manifestations, fever, and oral ulcers were less common. Consistent with these results, Naini et al. [9] studied the clinical manifestations of 55 patients with AAV vasculitis. The results of this study showed that Otitis and Mastoiditis are the most common clinical manifestations in GPA patients. In the present study, it was shown that hearing loss was the most common audiometry manifestation, which is in line with the study of Naini et al. that reported hearing loss was the most common audiometry manifestation. Rezaei Yazdi et al. [10] also studied the clinical manifestations of head and neck in 12 GPA patients. The results of this study showed that $72 \%$ of patients had complaints about head and neck pain and $65 \%$ had lower respiratory involvement. Recurrent sinusitis was seen to be present in $45 \%$ of the patients, nasal structure involvement in $72 \%$, ocular complaints in $27 \%$, and ear involvement in $45 \%$ of the cases.

In the current study, it was shown that increased thickness of the paranasal sinuses, pulmonary cavity, and pulmonary nodules are the most common radiological findings in GPA patients. Consistent with these results, Jiakai Li et al., in a study of 45 GPA patients, showed that cavitation was the most common radiological finding in those patients [11]. A recent study of 225 GPA cases showed that the presence of solid and pulmonary cavity nodules was associated with poorer prognosis in those patients [12]. In this regard, Hoffman et al. studied 158 cases of GPA to evaluate the clinical manifestations of GPA 
patients. They found that pulmonary involvement was present in $85 \%$ of GPA patients, of which $66 \%$ were pulmonary infiltrates, $58 \%$ were nodules, $30 \%$ were hemoptysis, and $28 \%$ were pleural effusion [13]. Cordier et al. studied radiologic and clinical aspects of 77 GPA patients. The results of this study showed that the pulmonary clinical manifestations of those patients included cough, dyspnea, hemoptysis, and chest pain. Fourteen patients had unilateral nodules, 39 patients had bilateral nodules, 15 patients had unilateral infiltrates, 26 patients had bilateral infiltrates, 9 patients had pulmonary opacity, and 3 patients had atelectasis [14].

C-ANCA positive and anti-PR3 positive were seen in $68.5 \%$ and $12.5 \%$ of GPA patients, respectively, and $8.33 \%$ of patients were simultaneously C-ANCA and anti-PR3 positive. Radice et al. studied the diagnostic value of PR3ANCA in GPA. The results of this study showed that new

\section{References}

1. Watts RA, Robson J. Introduction, epidemiology and classification of vasculitis. Best Pract Res Clin $\begin{array}{lll}\text { Rheumatol 2018; } & \text { 32(1):3-20. }\end{array}$ doi.org/10.1016/j.berh.2018.10.003.

2. Mossberg M. Studies of the pathophysiology and epidemiology of vasculitis. Lund University; 2017.

3. Watts RA, Mahr A, Mohammad AJ, Gatenby P, Basu N, Flores-Suárez LF. Classification, epidemiology and clinical subgrouping of antineutrophil cytoplasmic antibody (ANCA)-associated vasculitis. Nephrol Dial Transplant 2015; 30(suppl_1):i14-i22. doi.org/10.1093/ndt/gfv022.

4. Bessias N, Moulakakis KG, Lioupis C, Bakogiannis K, Sfyroeras G, Kakaletri K. et al. Wegener's granulomatosis presenting during pregnancy with acute limb ischemia. $\boldsymbol{J}$ Vasc Surg 2005; 42(4):800-04. doi: 10.1016/j.jvs.2005.05.038.

5. Leavitt RY, Fauci AS, Bloch DA, Michel BA, Hunder GG, Arend WP. et al. The American College of Rheumatology 1990 criteria for the classification of Wegener's granulomatosis. Arthritis Rheum 1990; 33(8):1101-07. doi: 10.1002/art.1780330807.

6. Nilsen AT, Karlsen C, Bakland G, Watts R, Luqmani R, Koldingsnes W. Increasing incidence and prevalence of ANCA-associated vasculitis in Northern Norway. Rheumatology (Oxford) 2020; 59(9):2316-24. doi: 10.1093/rheumatology/kez597.

7. Abdulkader R, Lane SE, Scott DG, Watts RA. Classification of vasculitis: EMA classification using CHCC 2012 definitions. Ann Rheum Dis 2013; 72(11):1888-88. doi: 10.1136/annrheumdis-2013-203511.

8. Berti A, Cornec D, Crowson CS, Specks U, Matteson EL. The Epidemiology of Antineutrophil Cytoplasmic Autoantibody-Associated Vasculitis in Olmsted County,
PR3-ANCA tests increase the accuracy of GPA diagnosis [15].

\section{Conclusion}

According to the findings of the present study, GPA was the most common type of vasculitis and ENT involvements were the most common clinical symptoms in patients with GPA. Due to the more common involvement of the upper airways, than other organs, and the referral of these patients to Amir Alam Center (Ear, Nose and Throat Treatment and Referral Center for Wegener patients with limited involvement-upper airways), the frequency of Wegener vasculitis seems to be higher than the findings of this study.

\section{Conflict of interest}

The authors report that they have no conflicts of interest to declare.
Minnesota: A Twenty-Year US Population-Based Study. Arthritis Rheumatol 2017; 69(12):2338-50. doi: 10.1002/art.40313.

9. Naini AS, Ghorbani J, Elahi SML, Beigomi M. Otologic manifestations and progression in patients with Wegener's granulomatosis: a survey in 55 patients. Iran $J$ otorhinolaryngol 2017; 29(95):327-31.

10. Rezaei Yazdi Z, Bagher beigi M. Investigation of head and neck clinical manifestations in wegner Journal Article.

11. Li J, Li C, Li J. Thoracic manifestation of Wegener's granulomatosis: Computed tomography findings and analysis of misdiagnosis. Exp Ther Med 2018; 16(1):41319. doi: $10.3892 /$ etm.2018.6154.

12. Russell B, Mohan S, Chahal R, Carette S, Pagnoux C. Prognostic significance of cavitary lung nodules in granulomatosis with polyangiitis (Wegener's): a Clinical Imaging Study of 225 Patients. Arthritis Care Res (Hoboken) 2018; 70(7):1082-89. doi: 10.1002/acr.23443.

13. Langford CA, Hoffman GS. Rare diseases.3: Wegener's granulomatosis. Thorax 1999; 54(7):629-37. doi: 10.1136/thx.54.7.629.

14. Cordier J-F, Valeyre D, Guillevin L, Loire R, Brechot J-M. Pulmonary Wegener's granulomatosis: a clinical and imaging study of 77 cases. Chest 1990; 97(4):906-12. doi: 10.1378/chest.97.4.906.

Radice A, Bianchi L, Maggiore U, Vaglio A, Sinico RA. Comparison of PR3-ANCA specific assay performance for the diagnosis of granulomatosis with polyangiitis (Wegener's). Clin Chem Lab Med 2013; 51(11):2141-49. doi: 10.1515/cclm-2013-0308. 
